\title{
Group velocity of acoustic waves in strongly scattering media: Dependence on the volume fraction of scatterers
}

\author{
M. L. Cowan, ${ }^{1}$ K. Beaty, ${ }^{1}$ J. H. Page, ${ }^{1}$ Zhengyou Liu, ${ }^{2}$ and Ping Sheng ${ }^{2}$ \\ ${ }^{1}$ Department of Physics and Astronomy, University of Manitoba, Winnipeg, Manitoba, Canada R3T 2N2 \\ ${ }^{2}$ Department of Physics, The Hong Kong University of Science and Technology, Clear Water Bay, Kowloon, Hong Kong
}

(Received 4 June 1998)

\begin{abstract}
We study, both experimentally and theoretically, the ballistic propagation of ultrasonic wave pulses through a random strongly scattering medium as a function of the volume fraction of the scatterers. The scattering medium consists of a liquid suspension of monodisperse glass beads, whose concentration is varied by controlling the upward flow of the liquid in a fluidized bed. At intermediate frequencies, where the acoustic wavelength $\lambda$ is comparable to the size of the glass bead scatterers, very strong scattering is observed, with the scattering mean free path reaching values as low as $\lambda / 2$. At high volume fractions of scatterers, the scattering results in pronounced dispersion, as demonstrated experimentally by the strong frequency dependence found in both the phase and group velocities. However, as the volume fraction is lowered, the dispersion is substantially reduced, in marked contrast to recent predictions for electromagnetic waves. Our experimental results are explained quantitatively by a theoretical model, based on a spectral function approach, that accounts for the renormalization of the scattering within the medium, an effect that is greatest when the concentration of scatterers is largest. The mechanisms underlying the frequency dependence of the velocities and their dependence on volume fraction are further elucidated by examining the ultrasonic energy density, both inside the scatterers and in the surrounding fluid. This allows us to show that the velocities are substantially slowed down both by (i) resonant scattering from the glass spheres, where energy is trapped within the solid scatterers, and by (ii) tortuosity effects, where the wave energy is largely confined to the tortuous fluid pathways. These results demonstrate convincingly why the phase and group velocities of acoustic waves vary strongly with frequency at high volume fractions of scatterers, but only show weak dispersive effects at low volume fractions. Furthermore, our microscopic picture of the dispersion gives a simple physical explanation of why its volume fraction dependence is opposite to that expected for light and other electromagnetic waves, where the velocity inside the scatterers is normally less than in the surrounding medium. [S1063-651X(98)10910-8]
\end{abstract}

PACS number(s): 43.35. $+\mathrm{d}, 43.20 .+\mathrm{g}, 62.30 .+\mathrm{d}$

\section{INTRODUCTION}

In recent years there has been renewed interest and progress in the study of the propagation of classical waves in strongly scattering inhomogeneous media [1,2]. For example, the realization that the diffusion approximation gives an excellent description of the propagation of multiply scattered classical waves has facilitated the investigation and explanation of a wide range of fascinating wave phenomena in strongly scattering materials; these include coherent backscattering, continuous-wave transmission, pulse propagation, and frequency, spatial, and temporal correlations [3-10]. Despite this progress, relatively little attention has been paid in such materials to the weak ballistic component of the total wave field that propagates through the medium without scattering out of the forward direction. In a pulsed experiment, this ballistic signal carries important information about the medium, including the behavior of the phase and group velocities $v_{p}$ and $v_{g}$, as well as the scattering mean free path $l_{s}$. Of particular interest is the behavior of the group velocity. In the intermediate frequency regime, where the wavelength is comparable to the size of the inhomogeneities, the strong scattering may lead to very large dispersion, with the possible result that the group velocity may exhibit anomalous behavior similar to that observed for optical propagation in homogeneous (on the scale of the wavelength) absorbing dielectric materials [11-19]. In fact, calculations of the group velocity for light propagation in a colloidal suspension of strongly scattering particles [20-22] predicted that the group velocity becomes strongly anomalous, exceeding the speed of light in vacuum over a substantial range of frequencies and supporting the idea that the group velocity completely loses its physical significance as the velocity of ballistic energy transport in such circumstances. However, recent measurements for acoustic waves [23] have shown that the group velocity can remain well defined even in the presence of strong resonant scattering, although the scattering does cause the velocity to vary strongly with frequency. These acoustic experiments were explained using a spectral function approach, which demonstrated how the group velocity could be accurately calculated when there is strong scattering due to shape resonances [23].

While these recent experiments might appear to have resolved these questions about the nature of the group velocity in the intermediate frequency regime of multiply scattering materials, important questions remain concerning the dependence of the group velocity on the volume fraction $\phi$ of the scatterers. The original calculations for light $[20,21]$ were done at low volume fractions, while the acoustic measurements were performed at high volume fractions. Recently it has been suggested that the pronounced dispersion predicted for electromagnetic waves at low volume fractions of scatterers becomes washed out at high volume fractions 
[2,24,25], possibly offering an explanation for the differences between the previous optical and acoustic results. One interesting implication of these predictions is that the group velocity for acoustic waves might have an even stronger frequency dependence at low volume fractions than at high volume fractions and could indeed exhibit anomalous behaviour after all.

In this paper we investigate this question both experimentally and theoretically by studying the ballistic propagation of acoustic wave pulses through a strongly scattering material as a function of the volume fraction of scatterers [26]. The scattering medium consists of monodisperse glass beads suspended in a liquid mixture of water and glycerol, where the impedance mismatch ratio for longitudinal acoustic waves has a large value of approximately 7 . The volume fraction of the glass beads was varied by fluidizing the suspension to counteract the sedimentation of the beads, allowing $\phi$ to be readily adjusted by controlling the flow rate. At high volume fractions of scatterers, pronounced dispersion is observed, consistent with previous measurements of the group and phase velocities in a similar system of randomly close-packed glass beads in water [23]. However, as the volume fraction is lowered, the variation of the velocities with frequency is progressively reduced, in marked contrast to the recent predictions for light $[2,24,25]$. We interpret these results using an effective medium model [23,27-29], based on a spectral function approach, in which each scattering unit is treated as an elastic sphere coated with a layer of liquid. This model shows how the substantial increase in the dispersion with volume fraction can be explained in terms of a renormalization of the effective medium in which the scatterers are embedded, an effect that becomes larger as the volume fraction $\phi$ increases. In contrast to the usual situation for light, the elastic wave velocities inside the scatterers are larger than in the surrounding fluid; thus it is possible to trap acoustic wave energy in the pockets of fluid between the glass bead scatterers, an effect that becomes especially pronounced at high volume fractions of scatterers when the fluid interstices become more enclosed. This effect is accurately captured by our coated sphere effective medium model, which gives an excellent description of the experimental data for the phase and group velocities, successfully elucidating the underlying physics of the strong volume fraction dependence of the dispersion resulting from acoustic shape resonances in the intermediate frequency regime.

\section{EXPERIMENT}

The volume-fraction dependence of ballistic transport was investigated experimentally using pulsed ultrasonic techniques because their ability to detect the field, rather than the intensity, allows the weak ballistic pulse to be detected even when it is buried in a much larger incoherent signal due to multiply scattered sound. To vary the volume fraction of scatterers in our system, we used a sample cell consisting of a fluidized bed, in which a $75 \%$ glycerol-25\% water mixture flowed upward to counteract the sedimentation of suspended glass beads. This choice of fluid was made to allow the beads to be conveniently suspended using modest flow velocities, while avoiding the unwanted absorption associated with very high fluid viscosities. The glass beads were monodisperse, with an average radius $a=0.438 \mathrm{~mm}$, having been sieved from a polydisperse distribution of spheres to limit the radii to a narrow range between 0.425 and $0.45 \mathrm{~mm}$. The material properties of the glass beads and fluid resulted in a very large mismatch in the acoustic impedance $\rho v$ since the speed of sound in the fluid is $1.84 \mathrm{~mm} / \mu \mathrm{s}$ and its density is 1.20 $\times 10^{3} \mathrm{~kg} / \mathrm{m}^{3}$, while the longitudinal velocity in the glass is $5.6 \mathrm{~mm} / \mu \mathrm{s}$, the transverse velocity in the glass is $3.4 \mathrm{~mm} / \mu \mathrm{s}$, and the density is $2.50 \times 10^{3} \mathrm{~kg} / \mathrm{m}^{3}$. Thus very strong scattering is expected in the intermediate frequency regime. Two sample cells were constructed, each with two plastic walls separated by metal spacers, which set the thickness $L$ of the samples to be either 7.76 or $12.2 \mathrm{~mm}$, corresponding approximately to 9 or 14 bead diameters, respectively. The liquid, pumped in from the bottom of the cell, passed through a diffuser to achieve an even flow across the cell. The fluid pump was driven by a variable speed electric motor, giving fine control over the flow rate. Since the settling speed of the beads depends on the volume fraction, changing the flow rate in the fluidized bed allowed $\phi$ to be easily varied over an extended range of values.

The measurements were performed in a large tank of water in order to provide efficient coupling between ultrasonic transducers and the sample. Several pairs of fairly wide-band 1-in.-diam plane-wave ultrasonic transducers were used, one transducer to generate the input pulse and the other to receive the transmitted signal. Each pair had a different central frequency, ranging from 1 to $5 \mathrm{MHz}$, allowing the samples to be studied for wavelengths ranging from 0.8 to 6 bead radii. The transducers were aligned along a common axis with the sample cell placed between them, in the far field of the generating transducer.

The transmitted wave form that was detected by the receiving transducer contained two components, a small ballistic pulse that consists of the unscattered or forward-scattered waves and a larger scattered component that is superimposed on the ballistic pulse at early times but also extends to much longer times as progressively longer and longer multiple scattering path lengths are traversed by the ultrasonic pulse [Figs. 1(a) and 1(b)]. To measure the group and phase velocities, we need to extract this ballistic pulse from the dominant multiply scattered waves. This can be achieved in ultrasonic experiments using piezoelectric transducers by taking advantage of the fact that the ballistic pulse is coherent, both spatially and temporally with the incident pulse, while, in contrast, the scattered waves arrive incoherently at the detector on account of the different path lengths traveled through the sample and the different angle-dependent phase shifts experienced on scattering. Since the transducer detects the average instantaneous field of the transmitted wave over the front face of the piezoelectric element, much of the scattered ultrasound is canceled in the detector due to the random phase fluctuations of the scattered waves across the transducer face, provided the area of the detector is larger than the speckle size (or coherence area). In our experiment, in which the detector is placed in the near field, the speckle size of the scattered waves is of order the square of the wavelength [6], so that, typically, several hundred speckles interfere destructively in the detector, giving good cancellation of the scattered component of the wave field and leaving the spatially coherent ballistic pulse unaffected. To further cancel scat- 


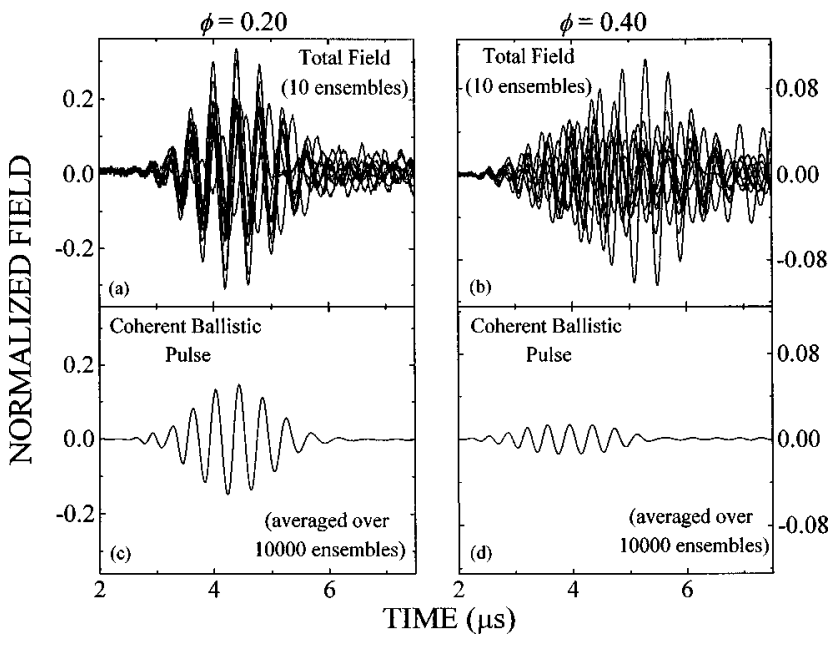

FIG. 1. Transmitted wave forms at volume fractions of 0.20 and 0.40 , for several representative bead configurations [(a) and (b), top], showing the superposition of the coherent (ballistic) and incoherent (scattered) components of the total field. The ballistic pulse is extracted by ensemble averaging the total fields over a large number of bead configurations, 10000 in this example [(c) and (d), bottom]. Here the central frequency in the pulse was $2.5 \mathrm{MHz}$ and the sample thickness was $L=7.76 \mathrm{~mm}$. At this frequency, the ratio of sample thickness to mean free path $L / l_{s}$ is 4.3 and 8.1 for $\phi=0.20$ and 0.40 , respectively.

tered sound we also averaged the detected signal over many repetitions of the input pulse using a digital oscilloscope. The beads were in continuous random motion, moving a mean distance that was negligibly small over the time the pulse takes to travel through the sample, but which was significant (appreciable compared to the wavelength) over the time between repetitions of the input pulse. Thus the average over many repetitions of the input pulse was in effect an average over many different ensembles of the scatterers and led to further cancellation of the scattered component of the transmitted sound, as is demonstrated in Fig. 1. In order to measure the full transmitted ultrasonic field for this demonstration, we replaced the 1-in.-diam detecting transducer with a miniature hydrophone, which had a size smaller than the ultrasonic wavelength, thereby avoiding phase cancellations across the face of the detector. In Figs. 1(a) and 1(b) we compare the detected fields at two volume fractions, respectively, by overlaying the detected fields for ten different ensembles of the scatterers. At the lower volume fraction of 0.20 where the scattering is weaker [Fig. 1(a)], evidence of the coherent component of the field can be seen by eye, as a substantial fraction of the detected field at early times (the first six or seven oscillations in the pulse) arrives in phase for each ensemble of the beads; the ballistic signal that is extracted by ensemble averaging the transmitted signal over 10000 realizations of the random configurations of the beads is shown in Fig. 1(c). When the volume fraction is increased to 0.40 , the true power of this configuration averaging technique becomes manifest. As a result of the increased scattering, the relative amplitude of the ballistic to scattered sound is decreased and a coherent component is not readily apparent [Fig. 1(b)]; however, after ensemble averaging the field, again over 10000 configurations of the beads, the scattered fields are effectively eliminated and the small ballistic signal

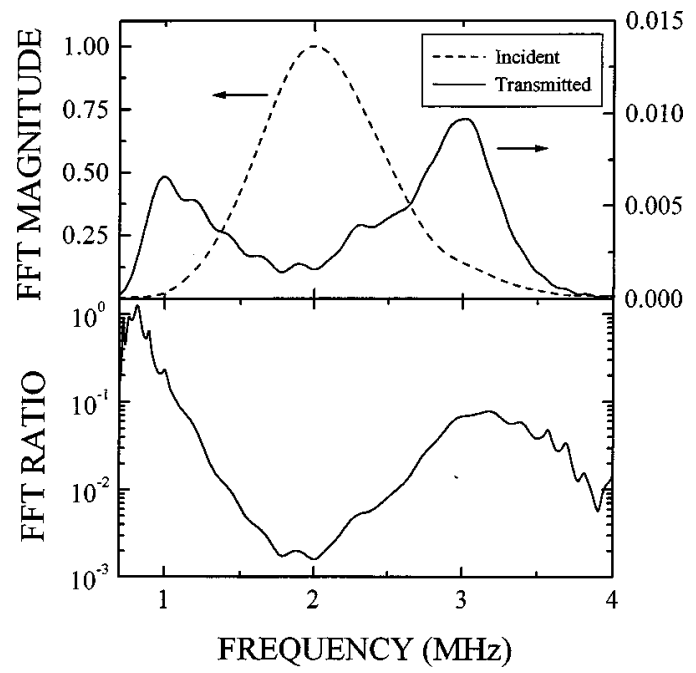

FIG. 2. The top panel shows the fast Fourier transforms (FFTs) of the input pulse and the ballistic pulse transmitted through a sample with $\phi=0.45$ and $L=7.76 \mathrm{~mm}$. The bottom panel shows the ratio of the transmitted to input FFTs, revealing a deep minimum in the transmission near $2 \mathrm{MHz}$ where the scattering is strongest.

clearly emerges with an excellent signal-to-noise ratio. Through the use of a large detector, further cancellation of scattered signal was achieved before ensemble averaging was performed.

Most of the experiments were performed using short input pulses, so that the frequency content of the pulse extended over the entire frequency spectrum of the transducer (approximately $50 \%$ of the central frequency). To measure the frequency dependence of the scattering mean free path, we first digitized the input pulse and the unscattered ballistic pulse, determined using the technique described above, and then compared the magnitudes of the fast Fourier transforms (FFTs) of the pulses as shown in Fig. 2(a) for a sample with a volume fraction of 0.45 and a thickness of $7.76 \mathrm{~mm}$. In Fig. 2(b) we show the ratio of the two FFTs, thus determining the magnitude of the ballistic signal over a bandwidth from about 1 to $3.5 \mathrm{MHz}$ by compensating for the frequencydependent response of the transducers. At low frequencies, the ratio is almost unity, showing that there is very little attenuation of the ballistic pulse, corresponding to the upper end in frequency of the weak Rayleigh scattering regime. However, as the frequency is increased above $1 \mathrm{MHz}$, the ratio drops precipitously, reaching a deep minimum at about $2 \mathrm{MHz}$ as a result of the strong scattering in the sample. The square of this ratio gives the frequency-dependent reduction in the transmitted intensity of the ballistic signal, from which the scattering mean free path $l_{s}$ was determined using the definition

$$
\frac{I}{I_{0}}=\exp \left[-L / l_{s}\right]
$$

where $I$ is the transmitted intensity, $I_{0}$ is the input intensity, and $L$ is the sample thickness. For the thicker sample, the corresponding FFT ratio disappeared into the noise level near the minimum at $2 \mathrm{MHz}$, requiring a different approach. In order to take transmission data in this region, we had to use a series of longer pulses, each with a narrow frequency 


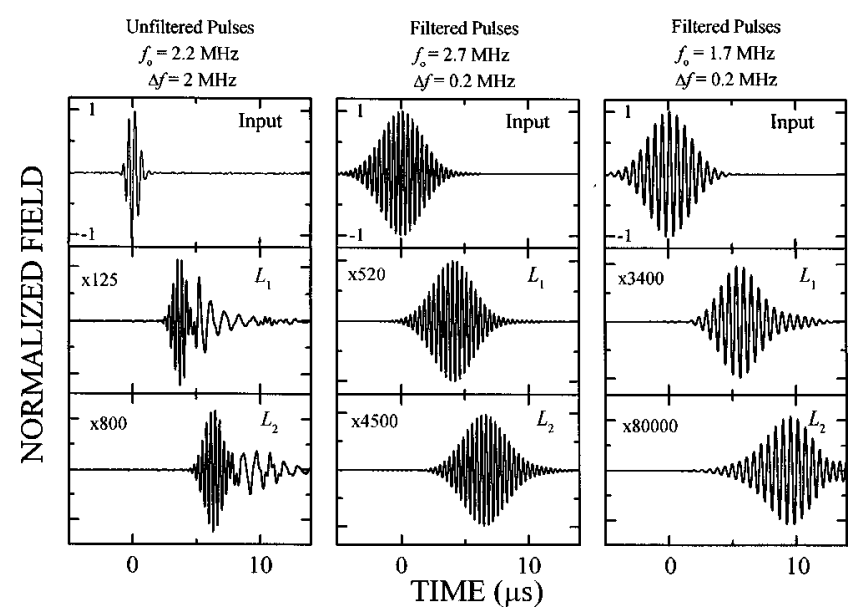

FIG. 3. The first column shows the unfiltered short input pulse and the resulting transmitted pulses through two sample thicknesses $\left(L_{1}=7.76 \mathrm{~mm}\right.$ and $\left.L_{2}=12.2 \mathrm{~mm}\right)$. The second and third columns show the result of digitally filtering these short pulses at two different central frequencies, indicated at the top of the figure.

bandwidth centered on the frequency of interest, thereby excluding from the pulse any large high or low frequency components that would have saturated the detection electronics at the increased sensitivity needed to measure these weak signals.

To measure the group and phase velocities, we used FFTs to digitally filter the detected pulses to form Gaussian pulses with a relatively tight bandwidth $(<10 \%)$ centered at several frequencies spanning the bandwidth of the pulse. An example of this technique is shown in Fig. 3 for data taken at a volume fraction of 0.45 , some of which is identical to that used to generate the FFTs shown in Fig. 2. The unfiltered short pulses in the first column show very strong dispersion effects, with a clear separation of faster high frequency components in the pulse from slower low frequency components, an effect that becomes more pronounced as the sample thickness increases and the pulse becomes broader and more distorted. It is interesting that these results are reminiscent of the effects predicted for electromagnetic pulse propagation in dispersive dielectrics with strong resonant absorption, as originally described using asymptotic methods by Sommerfeld [30] and Brillouin [31] and more recently by Oughstun and co-workers $[12,18]$. Evidently, the propagation of this short distorted pulse cannot be described by a single value of the group velocity. However, the frequency dependence of both the group and phase velocities can be accurately determined from these data by using a Gaussian filter function to narrow the frequency bandwidth of the pulse, so that dispersive distortion of the pulse is reduced and the group velocity description of pulse propagation is appropriate. The results of this filtering process for two central frequencies of 2.7 and $1.7 \mathrm{MHz}$ are shown in the second and third columns of Fig. 3 , respectively. Although small dispersion effects can still be seen in the slight broadening of the pulses with increasing sample thickness, the filtered pulses have a well defined central frequency and have symmetric peaks, so that both the phase and group velocities can be determined. The phase velocity was measured from the time taken by the carrier frequency oscillations to travel through both of the samples,

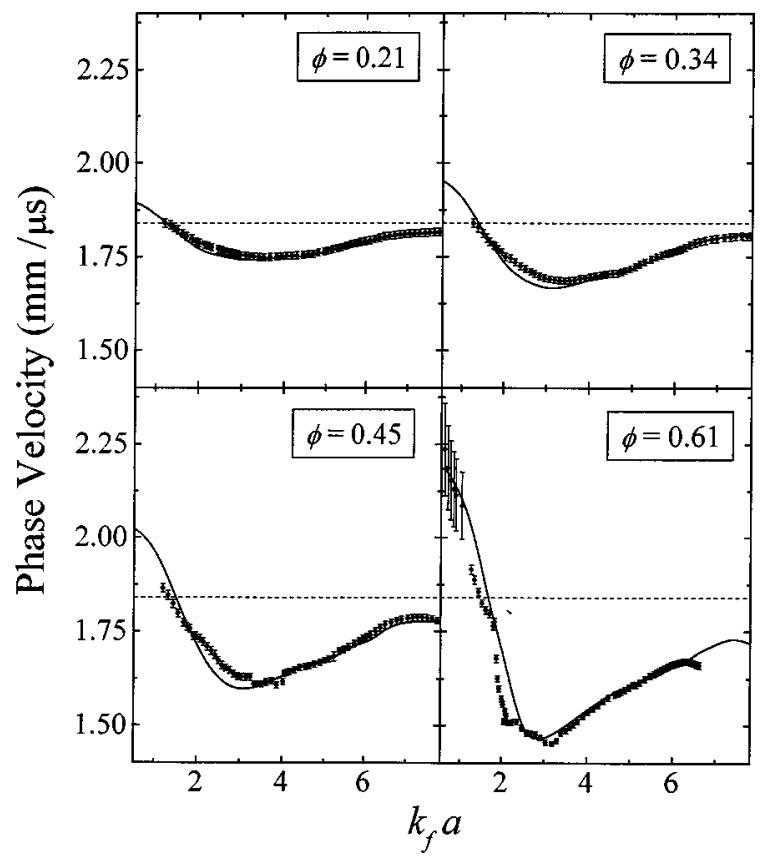

FIG. 4. Phase velocities $v_{p}$ at four different volume fractions, plotted against frequency normalized by the fluid velocity and bead radius. The data (symbols) are compared with the predictions of our effective medium theory (solid lines). The dashed line represents the velocity of sound in the bulk fluid.

using the condition that the velocity must be independent of sample thickness to resolve the possible ambiguity of one or more multiples of $2 \pi$ in the total phase shift of the transmitted pulse and hence to identify the phase velocity correctly. Simple inspection of Fig. 3 indicates that the pulses at 2.7 $\mathrm{MHz}$ travel faster than the $1.7-\mathrm{MHz}$ pulses, confirming that the group velocity is slower at the lower frequency. The group velocity was measured quantitatively by determining the envelopes of the incident and transmitted pulses, measuring the time interval between the peaks of the two pulse envelopes, and calculating the velocity from the ratio of sample thickness to pulse envelope propagation time.

\section{RESULTS AND DISCUSSION}

Using the experimental techniques described in the preceding section, we investigated the ballistic propagation of ultrasound through samples with volume fractions of glass beads ranging from 0.21 to 0.61 . Some of our measurements of the phase velocity at four volume fractions in this range are shown in Fig. 4. At the lowest volume fraction, the phase velocity is very close to the velocity of sound in the bulk fluid, indicated by the dashed line, over the entire frequency range. However, as the volume fraction is increased, a stronger frequency dependence develops, reaching a maximum variation with frequency of about $40 \%$ at $\phi=0.61$, which is about 7 times greater than at $\phi=0.21$. Our group velocity data (Fig. 5) show the same volume fraction trends, with relatively little dependence on frequency at the lowest volume fraction but with progressively larger variations in the frequency dependence of the velocity as the volume fraction increases. The variation of the group velocity is in general much larger than that of the phase velocity; for the most 


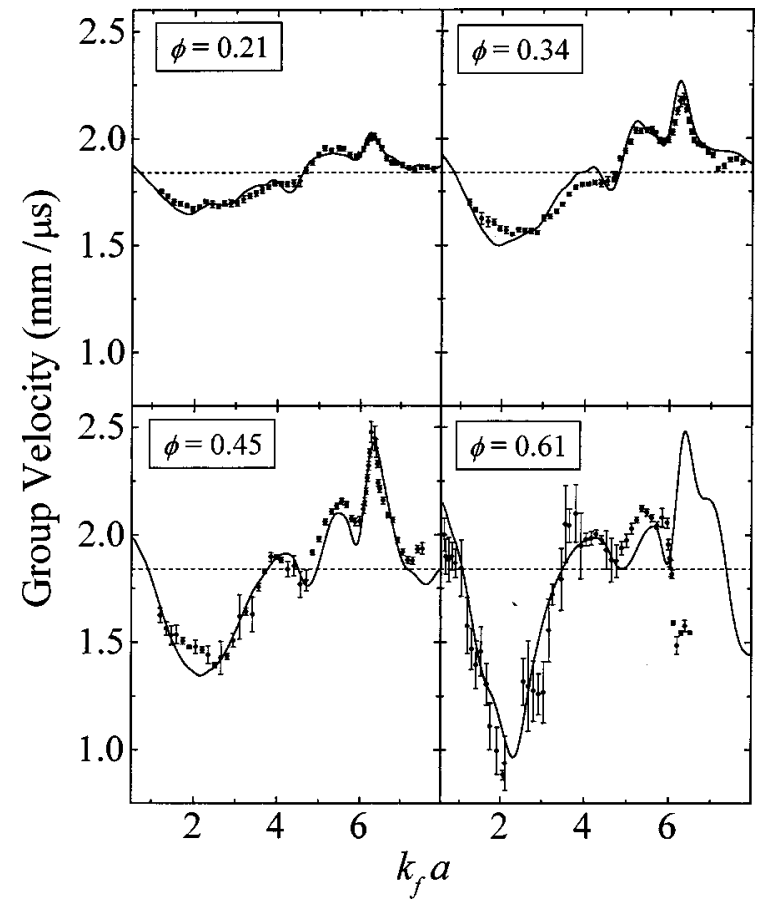

FIG. 5. Frequency dependence of the group velocities $v_{g}$ at four volume fractions, showing a comparison of the data (symbols) with our effective medium theory (solid lines). The dashed line represents the velocity of sound in the bulk fluid.

concentrated sample the group velocity changes by more than a factor of 2 , reaching values less than $1 \mathrm{~mm} / \mu$ s near the rather sharp minimum at $k_{f} a \approx 2-2.5$. Near this minimum, the group velocity is well below all the bulk velocities of both the solid and fluid constituents of the sample, suggesting that the scattering is having a strong effect on the ballistic propagation at the higher volume fractions. Measurements of the scattering mean free path (Fig. 6) shed further light on the connection between strong scattering and the propagation velocities. The mean free path has a pronounced dip for $k_{f} a$ between 2 and 3, which corresponds to the dips seen in both the phase and group velocities. This means that when scattering is at its strongest, the wave speeds of the ballistic pulse are greatly reduced. Careful in- spection of the figure shows that the position of the minimum in the mean free path moves to slightly lower frequencies as $\phi$ increases; the positions of the minima in the phase and group velocities also show the same trend, confirming that the reduction in the wave velocities is caused by scattering. The scattering mean free path data also indicate that the scattering in this system becomes very strong in this frequency range, as demonstrated by the fact that at the minimum in $l_{s}$ for $\phi=0.61$, the mean free path becomes smaller than one-half the wavelength of sound in the sample.

The results of these experiments show that despite the strong scattering, the group velocity is well behaved, exhibiting no anomalous properties such as negative or infinite values, over the entire range of frequencies and volume fractions investigated. One of the most striking features of our experimental results is the strong volume fraction dependence of both velocities. At low volume fractions there is weak dispersion, i.e., the velocities have little frequency dependence, but as the volume fraction is increased the dispersion increases dramatically. At high volume fractions this strong dispersion is similar to that found in our earlier experiments [23] on randomly close-packed $(\phi=0.63)$ glass beads in water. Our current results show clearly that the volume fraction dependence of the dispersion seen in this acoustic system is opposite to that predicted for electromagnetic waves $[2,24,25]$. Thus the idea that the most dramatic dispersion effects should be observed at low volume fractions of scatterers does not apply in acoustics, a result that we now explain theoretically using a simple spectral function approach.

To interpret these experimental results, we use a spectral function approach, which transcends the limitations of the traditional coherent potential approximation (CPA) and allows coherent wave propagation to be described quantitatively in the strongly scattering intermediate frequency regime where the wavelength is comparable to the size of the inhomogeneities [23,27-29]. To express this approach mathematically, let us first use the scalar wave case to illustrate the basic principles, although the actual calculations took fully into account the elastic nature of ultrasonic waves in the solid spheres, presented in the Appendix. The scalar wave equation may be written as

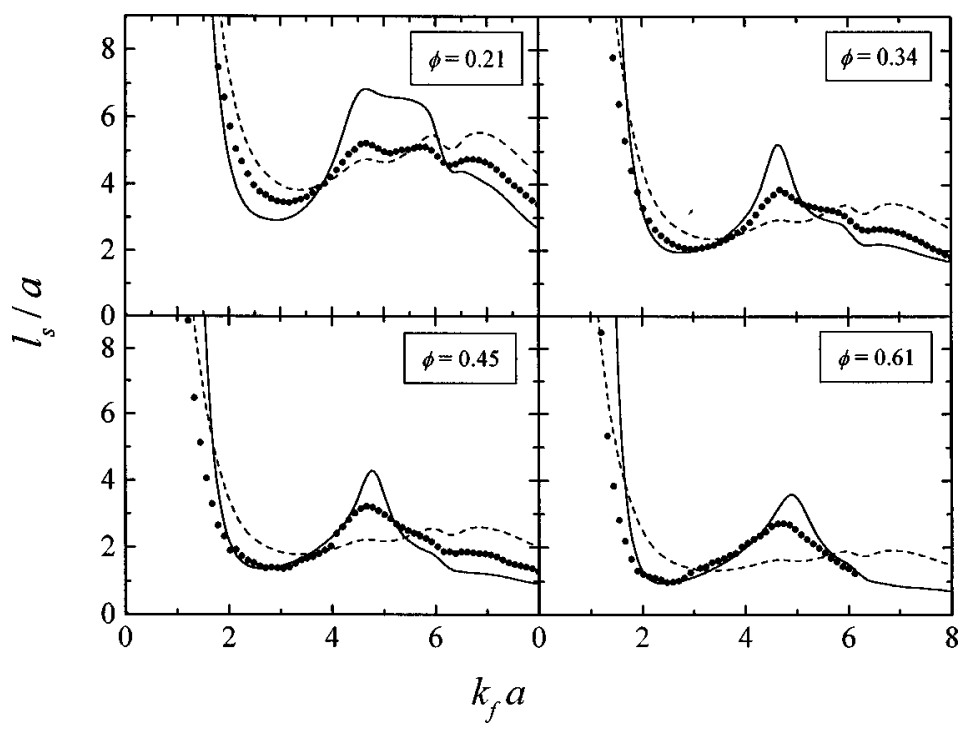

FIG. 6. Scattering mean free path $l_{s} / a$ at four volume fractions. The data (symbols) are compared with the predictions of the effective medium theory (solid lines) and with the calculation of $l_{s}$ from the scattering cross section of an isolated glass sphere in the fluid. 


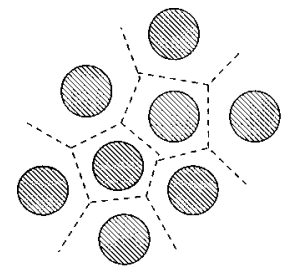

(a)

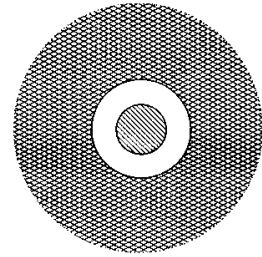

(b)
FIG. 7. In a random medium composed of a dispersion of spheres (a), the basic structural unit may be regarded as a coated sphere (b) embedded in a uniform medium of speed $v_{0}=\omega / k$.

$$
\left(\nabla^{2}+\frac{\omega^{2}}{v^{2}(r)}\right) \psi=0
$$

where $\psi$ denotes the wave amplitude and $v(r)$ is the local phase velocity. A slightly different way of writing the same equation is to add and subtract a constant term

$$
\left[\nabla^{2}+\frac{\omega^{2}}{v_{0}^{2}}-\left(\frac{\omega^{2}}{v_{0}^{2}}-\frac{\omega^{2}}{v^{2}(r)}\right)\right] \psi=0
$$

Here $v_{0}$ is an "effective medium", wave speed, which is treated as a dummy variable. One can write the configurationally averaged Green's function $G_{e}(\omega, \vec{k})$ as

$$
G_{e}(\omega, \vec{k})=\frac{1}{\omega^{2} / v_{0}^{2}-k^{2}-\Sigma_{v_{0}}(\omega, \vec{k})},
$$

where $\Sigma_{v_{0}}$ is the self-energy calculated relative to the reference medium speed $v_{0}$ and $\vec{k}$ is the Fourier transform variable of $\vec{r}$. To the leading order in the density of scatterers

$$
\Sigma_{v_{0}} \approx n t_{v_{0}}(0)
$$

where $n$ is the density of the scatterers and $t_{v_{0}}(0)$ $=f_{v_{0}}(0) / 4 \pi, f_{v_{0}}(0)$ being the forward-scattering amplitude. In a colloidal suspension, the inhomogeneous medium may be viewed as a collection of basic units consisting of coated spheres, shown in Fig. 7. The scattering problem is therefore that of a coated sphere embedded in a homogenized effective medium whose wave speed is given by $v_{0}$, identified at each $(\omega, \vec{k})$ point as $v_{0}=\omega / k$. The scattering amplitude can then be obtained as the solution to the corresponding boundary value problem, as discussed in the Appendix. Thus, from Eq. (5) we obtain the complex values of the self energy $\Sigma_{v_{0}}$ $=\Sigma_{\omega / k}(\omega)$ at each point in the $\omega$-k plane. Coupled with the condition that $v_{0}=\omega / k$, the Green's function is then given by $G_{e}(\omega, \vec{k})=-\Sigma_{\omega / k}^{-1}(\omega)$.

The traditional CPA condition for the determination of the effective medium velocity $v_{0}$ is that

$$
\operatorname{Re} \Sigma_{v_{0}} \cong n \operatorname{Re}\left[t_{v_{0}}(0)\right]=0
$$

However, in the strongly scattering intermediate frequency regime, $\Sigma$ becomes $k$ dependent. As a result, the solution of
Eq. (6) can either disappear, jump abruptly as $\omega$ varies, or become multivalued. In other words, the meaning of the CPA solutions becomes suspect. Instead of adopting this CPA condition, we find the excitations of the system by looking for the maxima of $-\operatorname{Im} G_{e}(\omega, \vec{k})=\operatorname{Im} \Sigma_{\omega / k}^{-1}(\omega)$, the socalled spectral function. Since the self-energy $\Sigma_{\omega / k}(\omega)$ is proportional to the forward-scattering amplitude, the peaks in the spectral function occur at the values of $\omega$ and $k$ when the scattering is a minimum and thus identify the propagating modes of the system. From Eq. (4) it can also be seen that the spectral function peaks at $\omega^{2}=v_{0}^{2} k^{2}$ when the CPA condition (6) is satisfied. Thus the spectral function approach reproduces the CPA in the low frequency regime where it is successful. Moreover, Im $\Sigma$ gives the width of the peaks. As $\operatorname{Im} \Sigma \rightarrow 0$ (true uniform medium), the peaks approach $\delta$ functions. In the intermediate frequency regime the peaks generally possess finite widths, implying that the modes identified by the spectral function peaks are quasimodes.

In Fig. 8 we show a contour plot of the spectral function in the frequency-wave-vector plane for two representative values of the glass-bead volume fraction. Note that there are no adjustable parameters in this calculation since the spectral function is determined by the scattering properties of a coated elastic sphere embedded in the effective medium; these are uniquely determined by the velocities and densities of the glass beads and fluid (given in Sec. II), the effective medium density [given by the average density of the medium $\left.\rho_{m}=\phi \rho_{\text {glass }}+(1-\phi) \rho_{\text {fluid }}\right]$, and the thickness of the fluid coating (determined by requiring that the volume of glass and fluid be conserved, so that $b / a=\phi^{-1 / 3}$, where $b$ is the radius of the coating). Since the peaks in the spectral function pick out the quasimodes with the least scattering, they form the dispersion curve, as shown for the two volume fractions illustrated in Figs. 7(a) and 7(b) by the solid white curves. In a pulse propagation experiment, the frequency (or more correctly the frequency spectrum) is set by that of the input pulse and the properties of the medium determine the corresponding wavelength of the excitation that propagates through the sample. Thus, to determine the dispersion curves that correctly describe the experimental situation from the spectral function, we use a peak-finding routine that scans the contour plots for the peak values of wave vector at each (constant) frequency [32]. The dispersion curves were then smoothed using a 25-point FFT smoothing routine to eliminate the high frequency "pixel" noise in the scanned positions of the peaks, arising from the finite resolution in $\omega$ and $k$ with which the spectral function was calculated. This procedure allows us to determine the true dispersion curve [33] with sufficient precision to reliably determine the propagation velocities from the spectral function.

We determine the phase velocity $v_{p}=\omega / k$ for each volume fraction of beads from the ratio of frequency to wave vector along the dispersion curve, giving the solid curves shown in Fig. 4. To account for the small 5\% variation in the size of the glass beads, the theory curves were also averaged over the bead size distribution. Excellent quantitative agreement with the data is found at all volume fractions, with the theory correctly predicting the magnitude of the phase velocity, as well as both the frequency and volume fraction dependence. The group velocity $v_{g}=d \omega / d k$ is calculated by numerically differentiating the dispersion curve to obtain the 

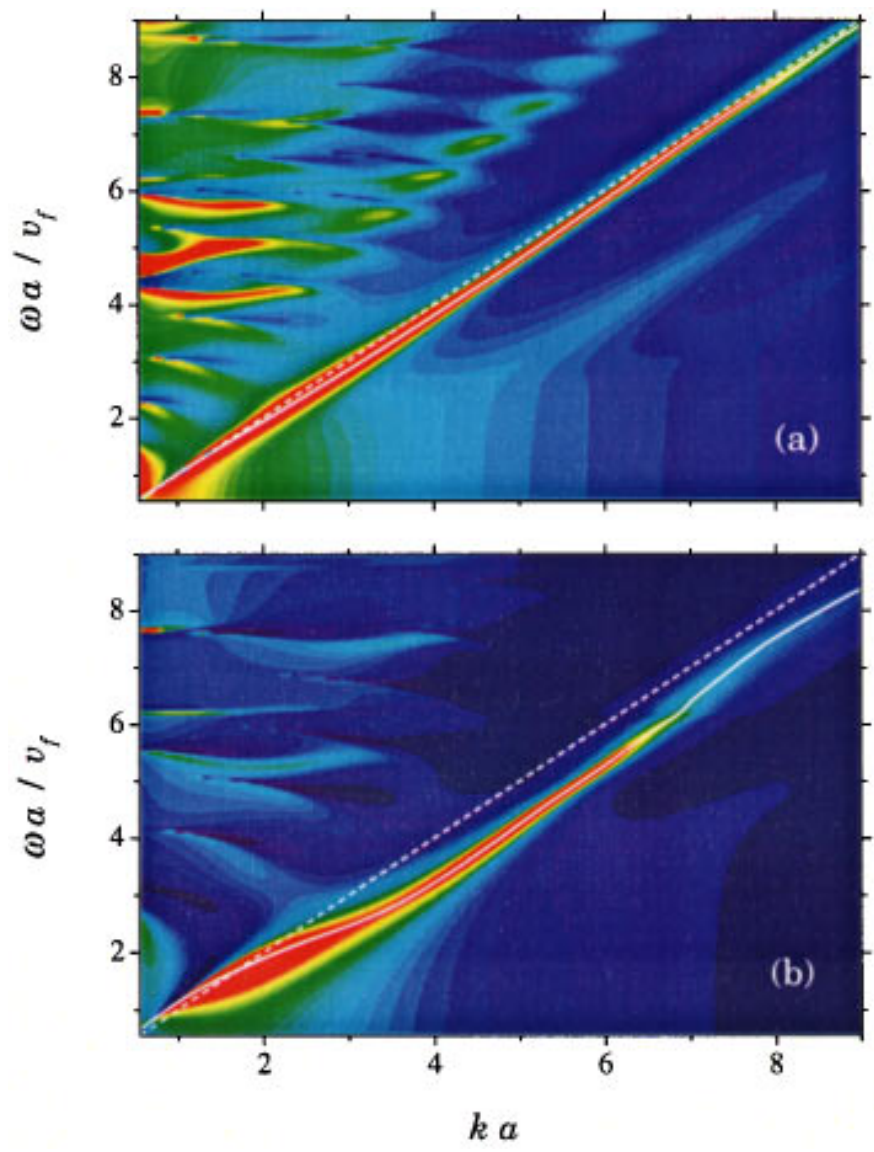

FIG. 8. (Color) Contour plot showing the spectral function at volume fractions of (a) $\phi=0.20$ and (b) $\phi=0.61$, as a function of normalized frequency $\omega a / v_{f}$ and effective medium wave vector $k a$. The magnitude of the spectral function is indicated by the colors, with red being high, yellow to green being intermediate, and blue being low. The dispersion curve (solid white line) is determined by the peaks in the spectral function and falls below the dispersion curve for the bulk fluid (dashed line) over most of the frequency range.

solid curves plotted in Fig. 5. Again, very good agreement with the data is found, with the considerable structure in the frequency dependence of the group velocity being correctly predicted at all frequencies and volume fractions investigated.

The same theoretical model can be used to estimate the scattering mean free path, which is calculated from the scattering cross section of the coated elastic sphere (see the Appendix). This was determined in two equivalent ways, either from the peak of the spectral function (which is equal to the imaginary part of the reciprocal of the self-energy) through the use of the optical theorem or by integrating the square of the scattering amplitude over all angles. Here the model is not as successful, as it underestimates the magnitude of the scattering because it assumes a uniform environment around each sphere, in contrast with the actual physical situation in which there are additional contributions to the scattering from the random configurations of the neighboring spheres. We account for this effect empirically by dividing the theoretical predictions by a phenomenological scaling parameter $p_{\sigma}$, which is taken to be independent of frequency and is obtained by fitting theory to experiment. This fitting param-

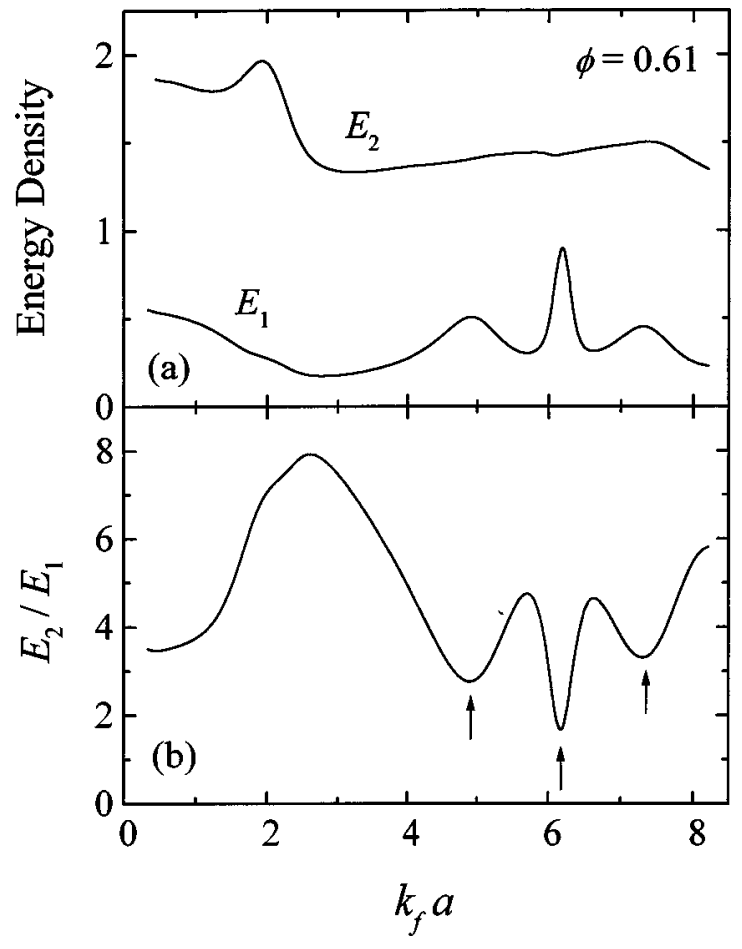

FIG. 9. (a) Average energy densities, normalized by the energy density of the incident wave, in the coated fluid region $\left(E_{2}\right)$ and in the solid core region $\left(E_{1}\right)$ plotted as a function of frequency. (b) Frequency dependence of the ratio $E_{2} / E_{1}$. The large peak at $k_{f} a$ $=2.6$ corresponds to the first minimum in the velocity dispersion and the physical situation where the wave energy is largely trapped in the interconnected fluid pathways. (See Fig. 10.) The arrows indicate the positions of the scatterer resonances. They correspond to the three successive minima in the velocity dispersions, seen most clearly in the group velocity data shown in Fig. 5.

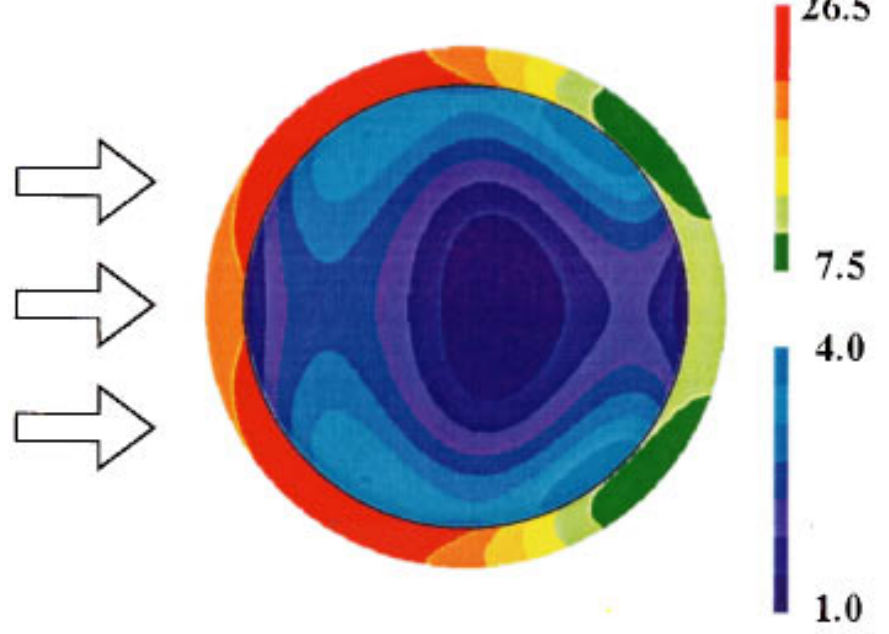

FIG. 10. (Color) Energy contour (in arbitrary units) in a single scatterer at frequency $k_{f} a=2.6$. The wave is incident on the coated sphere from the left, as indicated by the arrows. The magnitude is indicated by color, with red being the highest and blue being the lowest. Note that the energy density indicated by the upper scale (for the liquid coating) is almost 10 times that indicated by the lower (for the solid sphere). 
eter $p_{\sigma}$ is found to range from 1.6 to 2 as $\phi$ increases from 0.2 to 0.6 , consistent with exact multiple sphere scattering calculations that account explicitly for this additional scattering effect [28]. As shown by the solid curves in Fig. 6, reasonable agreement with the data is obtained, especially at high volume fractions, where the theory does an excellent job of predicting the location of the first minimum in the scattering mean free path. It is worth noting that the coated sphere calculation is much more successful in predicting the location of this minimum in $l_{s}$ than is a calculation based on the scattering cross section of single bare glass sphere in water, shown by the dashed curves. This demonstrates the power of our effective medium technique in accounting for the multiple scattering effects that are very important at high volume fractions.

To explain the strong volume fraction dependence of the velocities seen in the experiments, we calculated the average energy densities as a function of frequency both inside the glass beads (region 1) and in the fluid coating (region 2). This calculation is formulated in Appendix and the results at $\phi=0.61$ are shown in Fig. 9. The average energy densities, normalized by the energy density of the incident wave, are plotted in Fig. 9(a). This figure clearly demonstrates that the energy density in the fluid layer is much higher than in the solid spheres at all frequencies and delineates the structure in the frequency dependence that results from the scattering. While the normalized energy density in the fluid layer shows only a weak dependence on frequency at high frequencies, $E_{2}$ is seen to exhibit a maximum near $k_{f} a \approx 2$, corresponding to the resonant condition that the wavelength in the fluid layer $\lambda_{f}$ equals half the circumference of the solid sphere. As expected, this resonance weakens as the volume fraction decreases and the size of the fluid pockets between the beads increases. In contrast, the energy density in the glass beads $E_{1}$ exhibits three peaks at higher frequencies, which correspond to resonant scatterings by the solid spheres. By comparing Fig. 9(a) with Fig. 5, it can be seen that the peaks in $E_{1}$ correspond to the high frequency minima of the group velocity, indicating that strong resonant scattering can indeed slow down the propagation of acoustic waves. However, it should also be noticed that there is no resonant scattering in the spheres associated with the first and the largest dip in the group velocity. Instead, the energy density ratio $E_{2} / E_{1}$ exhibits a strong peak at that frequency, seen in Fig. 9(b), indicating that most of the wave energy is trapped in the fluid layer. This is clearly delineated in Fig. 10, where the energy density in a liquid-coated solid sphere, embedded in the renormalized effective medium, is plotted as a function of position for $k_{f} a=2.6$. In this frequency regime, the trapping of wave energy in the fluid causes wave propagation to slow down via a different physical mechanism: Here the slowdown is due to the tortuosity of the connected fluid pathways rather than resonant scattering by the solid spheres.

This microscopic picture of wave propagation in our acoustic system also provides the basis for understanding why the volume fraction dependence of the dispersion is opposite to that found for electromagnetic waves $[2,24,25,34]$. For electromagnetic waves, the velocity inside the solid scatterers is normally lower than in the surrounding medium since the scatterers usually have the higher dielectric constant. Consequently, in the vicinity of Mie resonances the electromagnetic energy density is very large inside the scatterers and small outside. At low $\phi$, this leads to a long dwell time of the waves inside the scatterers and large dispersion effects. However, as $\phi$ increases, the resonances become smeared out, as the wave energy that is predominantly concentrated in the scatterers couples from one scatterer to the next, and the dispersion is reduced [2,24,25]. In contrast, in our acoustic experiments the velocities of the elastic waves inside the solid scatterers are higher than in the surrounding medium, causing most of the wave energy to be concentrated in the fluid, as seen in Figs. 9 and 10. As a result, the two mechanisms that lead to wave dispersion in the acoustic case, tortuosity slowdown and resonant scattering in the spheres, are both more effective at high volume fractions of scatterers. It should be clear that the tortuosity effect is most pronounced at larger values of $\phi$ since the tortuosity of the fluid pathways increases with the volume fraction of scatterers as the pockets of fluid surrounding the scatterers shrink in size. Moreover, the effect of the resonant scattering in the spheres is to divert some of the wave energy from the fluid into the spheres, where the waves become partially trapped, thus also slowing down the propagation. However, for acoustic waves the majority of the wave energy still remains in the fluid and as a result the resonances do not become washed out at high volume fractions. As $\phi$ increases, the waves spend a greater fraction of their time partially trapped in the spheres simply because there are more of them; thus the magnitude of the dispersion increases correspondingly, in agreement with our experiments.

\section{CONCLUSIONS}

The good agreement between our theoretical model and experimental results suggests a physical picture for the wave propagation. In the intermediate frequency regime, the strong scattering causes the material properties of the medium to become renormalized, with the result that the effective medium in which the glass beads are embedded takes on some of the character of the scatterers themselves. Since it is precisely this renormalized effective medium that is sensed by the coherent ballistic wave, the phase and group velocities become significantly affected by the scattering; as a result, both velocities exhibit considerable dispersion, even though they describe the propagation of the small fraction of the incident pulse that is not scattered out of the forward direction. To model this behavior, we determine the dispersion curve using a spectral function approach, in which we calculate how the scattering properties of a coated elastic sphere are modified by the coupling to the embedding medium. Thus we are able to accurately calculate the renormalization of the effective medium and hence to correctly account for the frequency and volume fraction dependence of both the phase and group velocities. Furthermore, by examining the energy densities as a function of frequency, we identify two microscopic mechanisms for the velocity dispersion that is observed in our experiments: In addition to resonant scattering from the glass beads, where a significant fraction of the energy is trapped in the solid particles, we demonstrate the existence of "tortuosity slowdown," where the wave energy is mostly confined to the tortuous fluid pathways. Since the bulk of the acoustic energy remains in the fluid, both of these 
effects cause the dispersion to become greater as the volume fraction of the scatterers is increased, in agreement with our experimental results and with the quantitative predictions of our theoretical model.

\section{ACKNOWLEDGMENTS}

We thank NSERC, NATO, and RGC (Grant No. HKUST685/96P) for their support.

\section{APPENDIX: WAVE SCATTERING BY A LIQUID-COATED SOLID SPHERE IMMERSED IN AN EFFECTIVE LIQUID MEDIUM}

\section{Governing equations}

Consider a compressive wave incident along the $z$ direction on a coated elastic sphere embedded in a uniform effective medium, shown in Fig. 7. We denote the solid sphere as region 1 , the fluid coating as region 2 , and the outer effective medium as region 3. Azimuthal symmetry dictates that the displacements in the three regions may be expressed as

$$
\vec{u}^{i}=u_{r}^{i} \vec{e}_{r}+u_{\theta}^{i} \vec{e}_{\theta},
$$

where $\vec{e}_{r}, \vec{e}_{\theta}$, and $\vec{e}_{\phi}$ are unit vectors in spherical polar coordinates. The displacements $\vec{u}^{i}$, which satisfy the elastic wave equation in each region $i$, can be decomposed into longitudinal and shear parts by expressing the displacements in terms of the potential functions $\Phi_{i}$ and $\Psi_{i}$, where

$$
\vec{u}^{i}=\nabla \Phi_{i}+\nabla \times\left(\frac{\partial \Psi_{i}}{\partial \theta} \vec{e}_{\phi}\right) .
$$

Both $\Phi_{i}$ and $\Psi_{i}$ satisfy scalar wave equations, given by

$$
\nabla^{2} \Phi_{i}+\alpha_{i}^{2} \Phi_{i}=0
$$

and

$$
\nabla^{2} \Psi_{i}+\beta_{i}^{2} \Psi_{i}=0
$$

where $\alpha_{i}=\omega / c_{l}^{i}$ and $\beta_{i}=\omega / c_{t}^{i}$, with $c_{l}^{i}=\sqrt{\left(\lambda_{i}+2 \mu_{i}\right) / \rho_{i}}$ and $c_{t}^{i}=\sqrt{\mu_{i} / \rho_{i}}$ being the wave velocities of the longitudinal and transverse waves, respectively. $\lambda_{i}$ and $\mu_{i}$ are the Lamé constants, and $\rho_{i}$ denotes mass density. It should be noted that the $\Psi$ potential exists only in the solid core region, i.e., only $\Psi_{1} \neq 0$.

\section{Potential expansions in the three regions}

The field potential in the effective medium, region 3 , is composed of incident and scattered longitudinal waves. The incident plane wave can be expanded as

$$
\Phi_{3}^{(\text {inc })}=\Phi_{0} e^{i \alpha_{3} r \cos \theta}=\Phi_{0} \sum_{n=0}^{\infty}(2 n+1) i^{n} j_{n}\left(\alpha_{3} r\right) P_{r}(\cos \theta),
$$

where $j_{n}(x)$ is the $n$ th-order spherical Bessel function and $P_{n}(x)$ is the $n$ th-order Legendre polynomial. The scattered field potential may be expressed as

$$
\Phi_{3}^{\mathrm{sca}}=\sum_{n=0}^{\infty} A_{n} h_{n}^{(1)}\left(\alpha_{3} r\right) P_{n}(\cos \theta)
$$

where $h_{n}^{(1)}(x)$ is the spherical Hankel function of the first kind. The total potential in medium 3 is given by

$$
\Phi_{3}=\Phi_{3}^{\mathrm{inc}}+\Phi_{3}^{\mathrm{sca}}
$$

The field in the liquid coating layer (region 2) is also longitudinal. Thus only one potential is involved, i.e.,

$$
\Phi_{2}=\sum_{n=0}^{\infty}\left[B_{n} j_{n}\left(\alpha_{2} r\right)+C_{n} n_{n}\left(\alpha_{2} r\right)\right] P_{n}(\cos \theta),
$$

where $n_{n}(x)$ is the spherical Neumann function.

In the solid core, i.e., in region 1 , the $\Phi$ potential is given by

$$
\Phi_{1}=\sum_{n=0}^{\infty} D_{n} j_{n}\left(\alpha_{1} r\right) P_{n}(\cos \theta)
$$

while the $\Psi$ potential may be written as

$$
\Psi_{1}=\sum_{n=0}^{\infty} E_{n} j_{n}\left(\beta_{1} r\right) P_{n}(\cos \theta)
$$

The expansion coefficients $A_{n}, B_{n}, C_{n}, D_{n}$, and $E_{n}$ are determined by the boundary conditions

$$
\begin{aligned}
& \left.u_{r}^{(3)}\right|_{r=b}=\left.u_{r}^{(2)}\right|_{r=b}, \\
& \left.\tau_{r r}^{(3)}\right|_{r=b}=\left.\tau_{r r}^{(2)}\right|_{r=b}, \\
& \left.u_{r}^{(2)}\right|_{r=a}=\left.u_{r}^{(1)}\right|_{r=a}, \\
& \left.\tau_{r r}^{(2)}\right|_{r=a}=\left.\tau_{r r}^{(1)}\right|_{r=a}, \\
& \left.\tau_{r \theta}^{(1)}\right|_{r=a}=0 .
\end{aligned}
$$

The scattering amplitude $f(\theta)$ is determined from the set of coefficients $\left\{A_{n}\right\}$ and is given by

$$
f(\theta)=-\frac{i}{\alpha_{3} \Phi_{0}} \sum_{n=0}^{\infty} A_{n} e^{-i(n \pi / 2)} P_{n}(\cos \theta) .
$$

This is the basic equation used in the determination of the self-energy and the spectral function. The solution of these boundary value equations also allows the displacements in each region to be obtained from the potential functions, using Eq. (A2).

\section{Stress, strain, and energy}

Once the wave displacements are obtained using the method outlined in the preceding subsection, it is straightforward to determine the energy density. The strain tensor $\stackrel{\leftrightarrow}{\epsilon}^{i}$ may be expressed in terms of the displacement vector as 


$$
\stackrel{\leftrightarrow}{\epsilon}^{i}=\frac{1}{2}\left(\boldsymbol{\nabla} \vec{u}^{i}+\vec{u}^{i} \boldsymbol{\nabla}\right)
$$

and the stress tensor $\tau_{\zeta \xi}^{i}$ and the strain tensor are related by the law of elasticity

$$
\tau_{\zeta \xi}^{i}=\lambda_{i}\left(\epsilon_{r r}+\epsilon_{\theta \theta}+\epsilon_{\phi \phi}\right)+2 \mu_{i} \epsilon_{\zeta \xi}^{i} .
$$

The energy density $E_{i}$ is given by

$$
E_{i}=\frac{1}{2} \stackrel{\leftrightarrow}{\tau}^{i}: \stackrel{\leftrightarrow}{\epsilon}^{i}+\frac{1}{2} \rho_{i} \omega^{2} u_{i}^{2},
$$

where $\overleftrightarrow{\tau}: \overleftrightarrow{\epsilon}$ represents the scalar contraction of the stress and strain tensors.
[1] Scattering and Localization of Classical Waves in Random Media, edited by P. Sheng (World Scientific, Singapore, 1990).

[2] P. Sheng, Introduction to Wave Scattering, Localization, and Mesoscopic Phenomena (Academic, San Diego, 1995).

[3] M. P. van Albada and A. Lagendijk, Phys. Rev. Lett. 55, 2692 (1985).

[4] P. E. Wolf and G. Maret, Phys. Rev. Lett. 55, 2696 (1985).

[5] J. F. de Boer, M. P. van Albada, and A. Lagendijk, Phys. Rev. B 45, 658 (1992).

[6] J. H. Page, H. P. Schriemer, A. E. Bailey, and D. A. Weitz, Phys. Rev. E 52, 3106 (1995).

[7] H. P. Schriemer, M. L. Cowan, J. H. Page, P. Sheng, Z. Liu, and D. A. Weitz, Phys. Rev. Lett. 79, 3166 (1997).

[8] J. M. Drake and A. Z. Genack, Phys. Rev. Lett. 63, 259 (1989).

[9] G. Maret and P. E. Wolf, Z. Phys. B 65, 409 (1987).

[10] D. J. Pine, D. A. Weitz, P. M. Chaikin, and E. Herbolzheimer, Phys. Rev. Lett. 60, 1134 (1988).

[11] L. Brillouin, Wave Propagation and Group Velocity (Academic, New York, 1960).

[12] K. E. Oughstun and G. C. Sherman, Electromagnetic Pulse Propagation in Causal Dielectrics (Springer-Verlag, Berlin, 1994).

[13] C. G. B. Garrett and D. E. McCumber, Phys. Rev. A 1, 305 (1970).

[14] R. Loudon, The Quantum Theory of Light (Clarendon, Oxford, 1973).

[15] R. Loudon, in Diffuse Waves in Complex Media, NATO Advanced Study Institute, Series B: Physics, edited by J.-P. Fouque (Kluwer, Dordrecht, in press).

[16] S. Chu and S. Wong, Phys. Rev. Lett. 48, 738 (1982).

[17] R. L. Chiao, Phys. Rev. A 48, R34 (1993).

[18] K. E. Oughstun and C. M. Balictsis, Phys. Rev. Lett. 77, 2210 (1996).

[19] The behavior of the group velocity has been quite extensively studied in the context of optical propagation through homogeneous absorbing dielectric media in the vicinity of isolated exciton or polariton resonances [13-18]. There it has been shown both theoretically and experimentally that, for thin samples not more than several absorption lengths thick, the group velocity still describes the propagation of a pulse even when $v_{g}$ behaves so anomalously that $v_{g}>c, v_{g}= \pm \infty$, or $v_{g}<0[13,16]$. This unusual behavior results from the reshaping of the pulse due to the resonant absorption. However, in thicker samples, it is predicted that the pulse must eventually become so distorted that the concept of group velocity breaks down, necessitating the use of the energy velocity $v_{e}$, defined as the ratio of energy flux to energy density, to describe pulse propagation. It is nonetheless important to distinguish between the behavior of polariton resonances and shape (Mie) resonances, which correspond to the resonant scattering mechanism considered in this paper; the analogies between them are discussed in some detail in Ref. [22], although the coupling between resonant scatterers that is frequently very important in the intermediate frequency regime is not explicitly considered in this excellent review.

[20] M. P. van Albada, B. A. van Tiggelen, A. Lagendijk, and A. Tip, Phys. Rev. Lett. 66, 3132 (1991).

[21] B. A. van Tiggelen, A. Lagendijk, M. P. van Albada, and A. Tip, Phys. Rev. B 45, 12233 (1992).

[22] A. Lagendijk and B. A. van Tiggelen, Phys. Rep. 270, 143 (1996).

[23] J. H. Page, P. Sheng, H. P. Schriemer, I. Jones, X. Jing, and D. A. Wietz, Science 271, 634 (1996).

[24] K. Busch and C. M. Soukoulis, Phys. Rev. Lett. 75, 3442 (1995).

[25] K. Busch and C. M. Soukoulis, Phys. Rev. B 54, 893 (1996).

[26] For a brief preliminary account of this work see M. L. Cowan, K. Beaty, J. H. Page, Ping Sheng, and D. A. Weitz, Phys. Canada 53, 145 (1997).

[27] X. D. Jing, P. Sheng, and M. Y. Zhou, Phys. Rev. Lett. 66, 1240 (1991).

[28] X. D. Jing, P. Sheng, and M. Y. Zhou, Phys. Rev. A 46, 6513 (1992).

[29] P. Sheng, X. Jing, and M. Zhou, Physica A 207, 37 (1994).

[30] A. Sommerfeld, Ann. Phys. (Leipzig) 44, 177 (1914).

[31] L. Brillouin, Ann. Phys. (Leipzig) 44, 203 (1914).

[32] Note that when the spectral function is scanned at constant frequency, there are no spurious gaps in the dispersion curve even in the vicinity of the scattering resonances, unlike the situation when the spectral function is scanned at constant wave vector (as might be appropriate for interpreting other types of experiments such as Brillouin scattering); pseudogaps can appear in the spectral function when it is calculated from the scattering from a single coated sphere embedded in a uniform medium, but are not present in practice due to the random scattering from many neighboring spheres, an effect that has been confirmed theoretically using a more rigorous calculation that treats the scattering from the adjacent spheres exactly [28].

[33] Note that even when the scattering in our system is strongest, the real part of the complex wave vector is still significantly larger than the imaginary part, so that $\left(2 l_{\mathrm{ext}}\right)^{-2}=\left(2 l_{s}\right)^{-2}$ is negligible compared to $k^{2}$. Under these conditions, it can be shown rigorously (e.g., for the relatively simple case of dielectrics with absorption resonances) that the peaks in the spectral function, determined at constant frequency, give the dispersion curve that governs pulse propagation; thus the deviations when $k l_{\text {ext }}<1$ noted for polaritons in Ref. [22] are not relevant 
for interpreting our ultrasonic data. (In our system, the extinction length $l_{\text {ext }}$ is essentially equal to the scattering mean free path $l_{s}$ since the absorption length $l_{a} \gg l_{s}$, as determined by independent measurements of $l_{a}$ using diffusing ul- trasound [6].)

[34] A. Z. Genack, J. H. Li, N. Garcia, and A. A. Lisyansky, in Photonic Band Gaps and Localization, edited by C. M. Soukoulis (Plenum, New York, 1993), p. 23. 